\title{
Defect Creation in Crystals : A Portal to Directional Dark Matter Searches
}

\section{Kadribasic, Fedja}

2018-12

Kadribasic , F , Mirabolfathi , N , Nordlund , K , Holmström , E \& Djurabekova , F 2018 , '

Defect Creation in Crystals : A Portal to Directional Dark Matter Searches ' , Journal of Low

Temperature Physics , vol. 193 , no. 5-6 , pp. 1146-1150 . https://doi.org/10.1007/s10909-018-2062-5

http://hdl.handle.net/10138/308764

https://doi.org/10.1007/s10909-018-2062-5

cc_by_nd

acceptedVersion

Downloaded from Helda, University of Helsinki institutional repository.

This is an electronic reprint of the original article.

This reprint may differ from the original in pagination and typographic detail.

Please cite the original version. 
Fedja Kadribasic, Nader Mirabolfathi, Kai Nordlund, Eero Holmström, Flyura Djurabekova

\title{
Defect Creation in Crystals: A Portal to Directional Dark Matter Searches
}

the date of receipt and acceptance should be inserted later

\begin{abstract}
A large body of astrophysical observations indicate that around 85 percent of the matter in the universe is not made of recognized standard model particles. Understanding the nature of this so-called dark matter is of fundamental importance to cosmology, astrophysics, and high energy particle physics. We examine the response of commonly used semiconductor materials to low-mass WIMP interactions using numerical simulations based on classical interatomic potentials in these materials. These simulations, backed up by more precise density functional theory simulations and experiments, predict an angular dependence in the defect formation energy threshold that varies by around $20 \mathrm{eV}$ from minimum to maximum. They also predict a nonlinear energy loss that never produces phonons due to the nonzero energy required to form crystallographic defects. We argue that such nonlinear effects related to defect formation in single-electron resolution semiconductor detectors allows for very effective directional sensitivity and possible statistical nuclear recoil discrimination to dark matter signals for masses below $1 \mathrm{GeV} / c^{2}$.
\end{abstract}

Keywords Dark matter detectors, Particle dark matter, Semiconductors, Density functional theory, Molecular dynamics

Fedja Kadribasic, Nader Mirabolfathi

Department of Physics and Astronomy, Texas A\&M University,

4242 TAMU, College Station, TX 77840, USA

E-mail: fedjak@physics.tamu.edu, mirabolfathi@physics.tamu.edu

Kai Nordlund, Eero Holmström, Flyura Djurabekova

Helsinki Institute of Physics and Department of Physics, University of Helsinki,

Physicum, Kumpula campus, Gustaf Hällströmin katu 2,a FI-00560 Helsinki, Finland

E-mail: kai.nordlund@helsinki.fi, eero.holmstrom@gmail.com, flyura.djurabekova@helsinki.fi

Eero Holmström

COMP, Department of Applied Physics, Aalto University

PO Box 11100, 00076 Aalto, Finland

E-mail: eero.holmstrom@gmail.com 
A preponderance of indirect evidence indicates that dark matter makes up $\sim 85 \%$ of the matter in the universe ${ }^{1}$. Consequently, it has played a pivotal role in how baryonic structure has developed and evolved in the universe. However, it is difficult to speculate on the properties of this hypothetical substance since it has eluded direct detection for almost thirty years. Since Weakly Interacting Massive Particles (WIMPs) have been a prime candidate for dark matter, many experiments have been proposed and are running to search for dark matter in the mass and cross section parameter space where WIMPs could most likely be found. However, these experiments are poised to reach the so-called neutrino floor that presents a background that cannot be discriminated from the dark matter interaction signal.

It may be possible to circumvent this background with a directional dark matter detector since this could discriminate signal from background based on direction. However, directionality comes at a serious price for low rate particle detection experiments. For example, time-projection chamber experiments using low pressure gases, which can discriminate on an event-by-event basis, require enormous volumes to have exposures high enough to detect dark matter ${ }^{2}$. Another hurdle for dark matter direct detection is that the dark matter spectrum is completely feature-less, which makes discriminating signal from noise challenging. There has been a lot of progress recently on phonon-mediated detectors, especially those using Neganov-Luke phonon amplification ${ }^{3}$, which could lead to large-mass semiconductor detectors with single-electron resolution ${ }^{4}$. Consequently, we propose to use the process of defect creation in single-crystalline semiconductors, such as germanium $(\mathrm{Ge})$ and silicon $(\mathrm{Si})$, used for detectors to possibly address both problems since the crystal direction dependent energy threshold for defect creation could be used for directional sensitivity and the energy loss due to defect creation could cause features in the expected recoil energy spectrum.

Currently, no computer models or experimental data can predict the minimum energy required for electron-hole pair production. However, ample evidence indicates that Lindhard theory breaks down below $\sim 100 \mathrm{eV}$, and computational evidence indicates that the energy for electron-hole pair production should be at least proportional to the energy needed for defect formation ${ }^{5,6,7,8,9,10,11,12}$. For this reason and for simplicity, we assume that the energy threshold for electronhole pair formation is equivalent to that for Frenkel defect formation in this paper. Rather than perform a density functional theory (DFT) time-dependent calculation, which would be too computationally-intensive to find the angular dependence of the energy threshold, we perform molecular dynamics simulations using classical potentials that are a good approximation to the full results ${ }^{13}$. We use the Stillinger-Weber potential since it provides the results closest to the full DFT calculations ${ }^{14,15}$. Similar numerical calculations yield the energy never transferred to phonons, which we refer to as the energy lost since the detector measures the total energy using the phonon signal, given a range of nuclear recoil energies.

The Frenkel pair defect creation thresholds found using these numerical calculations are shown in Fig. 1 via polar projection plots for Ge (top) and Si (bottom). The plots show the upper hemisphere of the defect creation surface as a function of recoil angle in detector coordinates assuming the Stillinger-Weber potential for both $\mathrm{Ge}$ and $\mathrm{Si}$. The lower hemisphere is not shown since, in principle, the thresholds are symmetric with respect to reflections across the [100]-[010] plane. The 


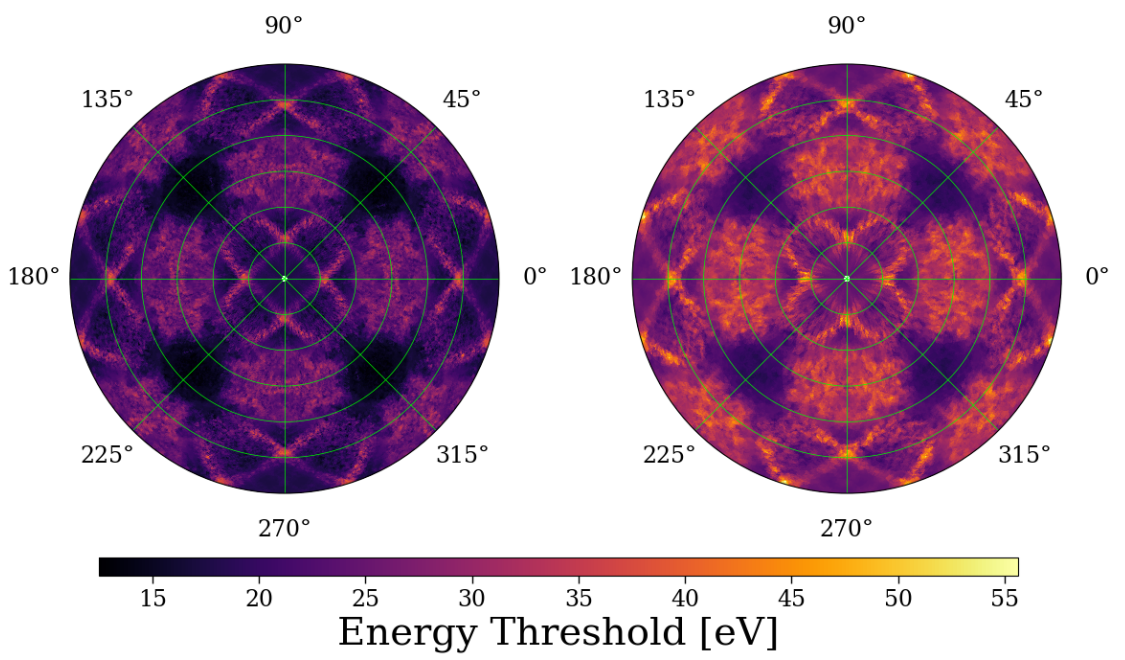

Fig. 1 Polar projections showing the energy thresholds for Ge (left) and Si (right) assuming the Stillinger-Weber potential calculated using molecular dynamics simulations. Only the upper hemisphere is shown since the thresholds are symmetric with respect to reflections across the [100]-[010] plane. The range of energy thresholds is $12.8-40 \mathrm{eV}$ for $\mathrm{Ge}$ and $18-55.6 \mathrm{eV}$ for $\mathrm{Si}$.

range of energy thresholds is $12.8-40 \mathrm{eV}$ for $\mathrm{Ge}$ and $18-55.6 \mathrm{eV}$ for $\mathrm{Si}$. We simulate 84,938 events for Ge and 24,157 for Si.

The average energy loss due to defect formation over all recoil directions simulated is shown in Fig. 2 on the left. We simulate 6714 events randomly distributed on the unit sphere in Ge for nuclear recoil energies ranging from 2 to $200 \mathrm{eV}$ inclusive. It shows how, at low recoil energies, the energy lost takes on discrete values that, at higher energies, get smoothed out into a linear fit. These energy loss steps end up corresponding to peaks in the expected measured recoil energy spectrum as we discuss later. To simulate this effect, we model the energy loss with a linear fit to the mean data with a jump at $40 \mathrm{eV}$, as shown on the right of Fig. 2. The line is fit using the mean data values above $100 \mathrm{eV}$.

Calculating the total signal rate to see the modulation and the expected recoil energy spectrum to see the effect of the energy loss requires performing integrals over the dark matter differential rate per unit steradian per unit recoil energy for a given dark matter mass and detector material. Ref. ${ }^{16}$ gives this differential rate as

$$
\frac{\partial^{2} R}{\partial E_{r} \partial \Omega_{r}}=\frac{\rho_{0} \sigma_{\chi-n} A^{2}}{4 \pi m_{\chi} \mu_{\chi n}^{2}} \times F^{2}\left(E_{r}\right) \hat{f}_{\mathrm{lab}}\left(v_{\min }, \hat{\boldsymbol{q}}_{r} ; t\right)
$$

where $m_{\chi}$ is the WIMP mass, $\mu_{\chi n}$ is the WIMP-nucleon reduced mass, $\rho_{0}=$ $0.3 \mathrm{GeV} \mathrm{cm}^{-3}$ is the local dark matter density, $A$ is the mass number of the nucleus, $\sigma_{\chi-n}$ is the WIMP-nucleon cross section, $v_{\min }=\sqrt{2 m_{N} E_{r}} / 2 \mu_{\chi n}$ is the minimum WIMP speed required to produce a nuclear recoil of energy $E_{r}$ for a given nuclear mass $m_{N}$, and $F^{2}\left(E_{r}\right)$ is the Helm nuclear form factor ${ }^{17}$. 

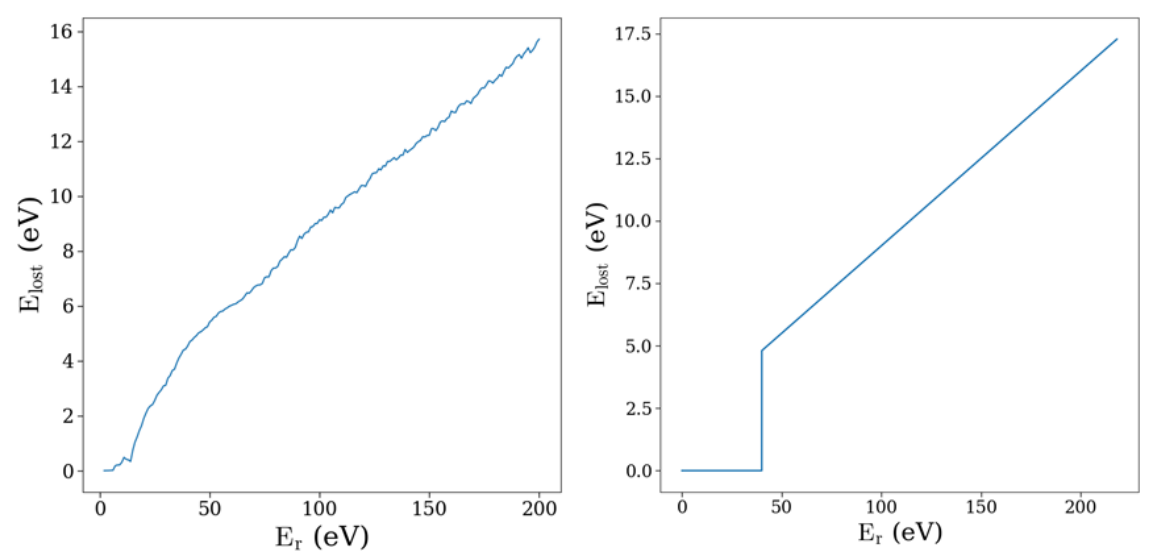

Fig. 2 Plots showing the mean energy lost to defect creation over all simulated directions for Ge from numerical simulations (left) and the model that we use in this work to simulate the effect it could have on the recoil energy spectrum (right). Every change in slope in the energy loss could cause a peak in the nuclear recoil energy spectrum, so we assume a linear relation with a discontinuous jump at $40 \mathrm{eV}$ from $0 \mathrm{eV}$ to simulate this effect.

The Radon transform of the WIMP velocity distribution $f_{\text {lab }}(v)$ is given by ${ }^{16}$ as

$$
\hat{f}_{\mathrm{lab}}\left(v_{\min }, \hat{\boldsymbol{q}} ; t\right)=\frac{1}{N_{\mathrm{esc}} \sqrt{2 \pi \sigma_{v}^{2}}}\left[\exp \left(-\frac{\left|v_{\min }+\hat{\boldsymbol{q}} \cdot \boldsymbol{v}_{\mathrm{lab}}\right|^{2}}{2 \sigma_{v}^{2}}\right)-\exp \left(-\frac{v_{\mathrm{esc}}^{2}}{2 \sigma_{v}^{2}}\right)\right]
$$

where $\hat{\boldsymbol{q}}$ is the recoil direction in detector coordinates, $\boldsymbol{v}_{\text {lab }}$ is the velocity of the laboratory relative to a stationary observer, $v_{\mathrm{esc}}$ is the circular escape velocity at the Solar System's distance from the Milky Way's center, $\sigma_{v}=v_{0} / \sqrt{2}$ is the dark matter velocity dispersion, and $N_{\text {esc }}$ is a normalization factor. We use $v_{0}=220 \mathrm{~km} \mathrm{~s}^{-1}$ for the circular speed and $v_{\mathrm{esc}}=544 \mathrm{~km} \mathrm{~s}^{-116}$. We use Appendix B of ${ }^{18}$ to find the total lab velocity using the galactic rotation, solar motion, Earth's revolution, and Earth's rotation contributions. We assume the detector is located at SNOLAB $\left(46.4719^{\circ}, 81.1868^{\circ}\right)$, and we calculate the differential rate on September 6, 2015 to coincide with Ref. ${ }^{16}$.

For any dark matter experiment, the total integrated rate is limited by the minimum energy threshold of detection below which the detector cannot observe an event. For very high resolution phonon-mediated charge detectors, this energy threshold is equivalent to that required to form electron-hole pairs. Since we expect the energy threshold for Frenkel defect formation to be proportional to that for electron-hole pair creation, setting the energy thresholds in Fig. 1 as the lower limit to the total rate integral as an approximation gives the expected modulation at a given dark matter mass. Mathematically,

$$
R(t)=\oint_{4 \pi} \int_{E_{t h}(\theta, \phi)}^{E_{r}^{\max }} \frac{\partial^{2} R}{\partial E_{r} \partial \Omega_{r}} \mathrm{~d} E_{r} \mathrm{~d} \Omega_{r} .
$$

Combining equations 3,1 , and 2 with the energy thresholds shown in Fig. 1 yields an integrated rate with a strong diurnal modulation for certain dark matter 


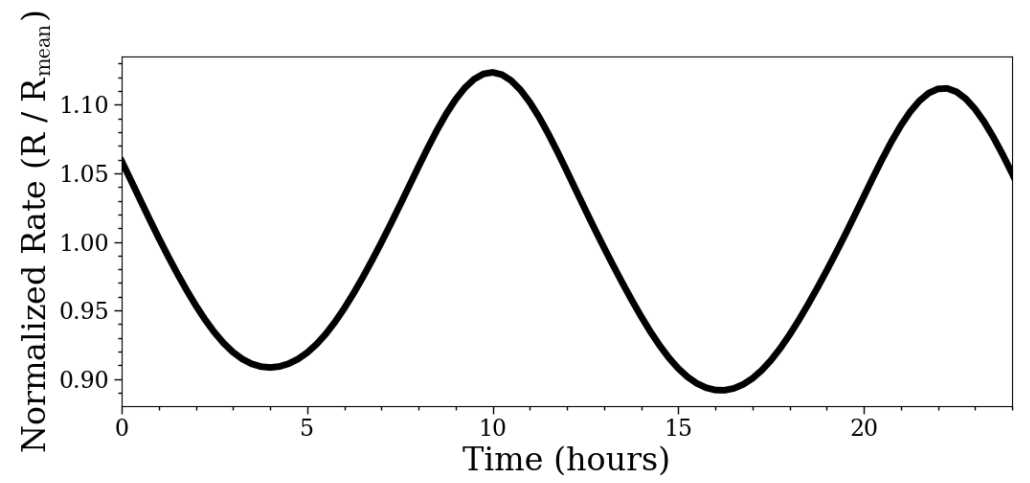

Fig. 3 Normalized expected integrated rate with respect to mean over one day given $240 \mathrm{MeV} / \mathrm{c}^{2}$ dark matter interacting with a Si detector and assuming an electron-hole pair energy threshold equal to that for Frenkel defect formation. We assume a nucleon-WIMP cross section of $10^{-39} \mathrm{~cm}^{2}$ for a detector at the SNOLAB site on September 6, 2015.

masses. As shown in Fig. 3, a Si dark matter detector looking for $240 \mathrm{MeV} / \mathrm{c}^{2}$ dark matter would see a nearly sinusoidal modulation of $\sim 10 \%$ from the mean value over half a day. The energy threshold causes the expected total signal to drop significantly at low dark matter masses, but it also causes very strong signal modulation. As the dark matter mass increases, the amount of modulation decreases since the overall signal is dominated by high recoil energy events not affected by the energy thresholds. Consequently, there is an optimal region of dark matter parameter space where the modulation is reasonably large but the overall rate has not been reduced significantly.

The presence of this effect could have a widespread impact on the field of dark matter direct detection experiments, the most straightforward of which is the daily modulation that can be used as a signature of dark matter. Related to this is the fact that it could be used to discriminate dark matter from the otherwise indistinguishable solar neutrino signal, which should have a diurnal variation as well but a different phase. Additionally, because the strength of the integrated rate modulation is a function of the dark matter mass, this effect could provide another handle to set limits on dark matter mass.

To find the effect of the energy loss, we perform integral 3 to find the differential rate per unit recoil energy, which gives the differential rate one assumes for a perfect detector. We choose $10^{8}$ sample events from the distribution given by $\frac{\partial^{2} R}{\partial E_{r} \partial \Omega_{r}}$ and add energies sampled from a gaussian distribution centered at $0 \mathrm{eV}$ with standard deviation of $1 \mathrm{eV}$. This result is integrated over all angles using Eq. 3 to find the blue curve on the leftmost plot in Fig. 4, which gives the expected differential rate assuming the detector has $1 \mathrm{eV}$ recoil energy resolution. We take the energy loss into account by sampling the events from the previous step and subtracting the mean energy loss to yield the orange curve on the left with the peak. Performing a similar calculation for more dark matter masses gives the curves on the right. 

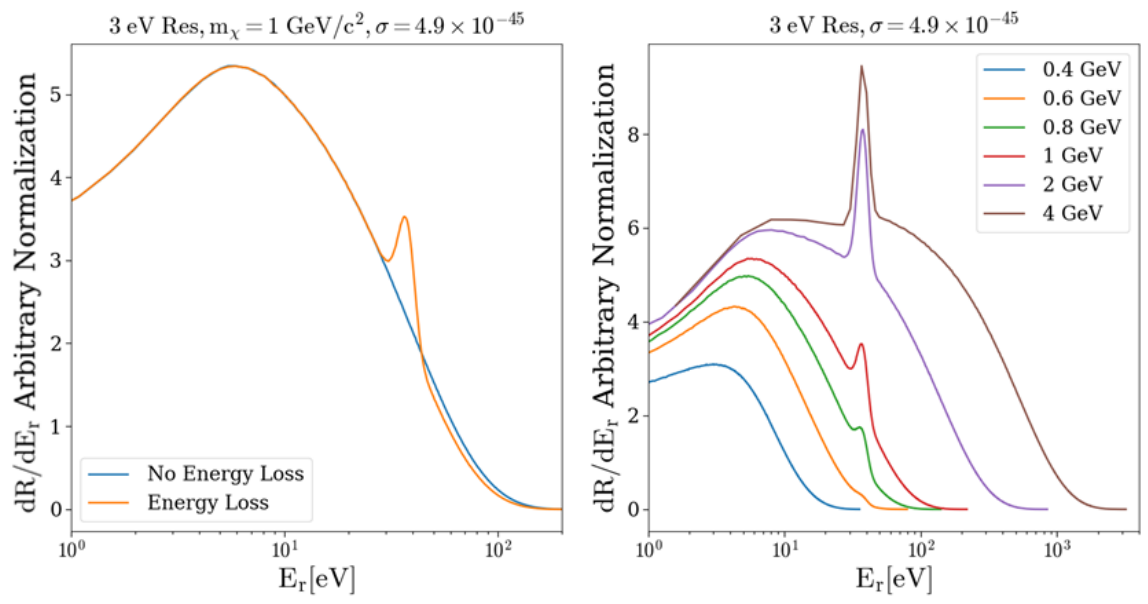

Fig. 4 Differential rate assuming a detector with $1 \mathrm{eV}$ energy resolution with and without energy loss for $1 \mathrm{GeV} / c^{2}$ dark matter (left) and for several other masses (right). The effect of the losses is that, at a given energy, a detector measures both the events that have a particular recoil energy and no energy loss and those with a higher recoil energy that have lost energy to defect formation. Consequently, the peak in recoil energy corresponds to the $40 \mathrm{eV}$ jump we assume to model this effect. Monte Carlo sampling is used to find the effects of the energy resolution and energy loss.

Fig. 4 shows the effect that the phonon energy loss can have on the dark matter spectrum at low recoil energies. Applying the energy losses transforms the flat, featureless spectrum to one with a peak that corresponds to the energy at which higher potential energy defects start to occur, as shown in the plots in Fig. 2. The change in the differential rate spectrum further emphasizes the importance of developing detectors that can probe ever lower energy thresholds since doing so not only probes large regions of dark matter parameter space but also accentuates the features in Fig. 4. Nonetheless, despite the potential impact this method could have on the field of direct dark matter detection, the change in spectra can most likely only be observed once many events have been measured. This limits the applicability of this method to future dark matter experiments that will, hopefully, have enough statistics to see this effect. However, it could still be extremely useful for other kinds of experiments, such as those attempting to detect CENNS like the MINER experiment.

There are many research areas where this idea can be applied that range far beyond simply signal-background discrimination in semiconductor dark matter detectors. For example, since this effect is independent of the charge signal, this method opens up many different kinds of detector materials to use for detecting dark matter, such as sapphire. Additionally, if materials exist that have an even larger range of energy losses depending on direction, it might be possible to have directional detectors utilizing this method via energy binning. This idea would then synergize with many current CENNS experiments that expect much larger event rates than dark matter, such as the MINER experiment at Texas A\&M University, which is poised to use the semiconductor detectors considered in this work. It could even act as a cross-disciplinary probe of DFT calculations since, at the moment, there is no strong experimental evidence at the atomic level to 
support their results, which are crucial for developing materials resistant to high radiation levels.

In conclusion, we have demonstrated an idea for a possible directional phononmediated charge detector using the anisotropic energy threshold for electron-hole pair production. For simplicity, in this study we assume that the energy threshold for Frenkel pair creation is equal to that for defect production and calculated the expected modulation in the total integrated dark matter rate as a function of dark matter mass. Additionally, we have presented a method that uses the energy lost to defect creation during nuclear recoil events in dark matter detectors to potentially discriminate nuclear recoil signal from electron recoil backgrounds. The potential impact of these results motivates research into novel detector materials and thorough calibration thereof.

Acknowledgements N. M. acknowledges Mitchell Institute For Fundamental Physics financial support. E. H. acknowledges financial support from the Emil Aaltonen foundation and the Academy of Finland through the Centres of Excellence Program (Project No. 251748). 


\section{References}

1. P. A. R. Ade et al. (Planck), Astron. Astrophys. 571, A16 (2014), arXiv:1303.5076 [astro-ph.CO] .

2. F. Mayet et al., Phys. Rept. 627, 1 (2016), arXiv:1602.03781 [astro-ph.CO] .

3. P. N. Luke, J. Beeman, F. S. Goulding, S. E. Labov, and E. H. Silver, Nucl. Instrum. Methods A289 (1990) 406-409., Nucl. Instrum. Meth. A289, 406 (1990).

4. N. Mirabolfathi, H. R. Harris, R. Mahapatra, K. Sundqvist, A. Jastram, B. Serfass, D. Faiez, and B. Sadoulet, Nucl. Instrum. Meth. A855, 88 (2017), arXiv:1510.00999 [physics.ins-det] .

5. J. E. Valdes, C. Parra, J. Diaz-Valdes, C. D. Denton, C. Agurto, F. Ortega, N. R. Arista, and P. Vargas, Phys. Rev. A 68, 064901 (2003).

6. S. N. Markin, D. Primetzhofer, and P. Bauer, Physical Review Letters 103, 113201 (2009).

7. D. Primetzhofer, Phys. Rev. B 86, 094102 (2012).

8. A. Lim, W. M. C. Foulkes, A. P. Horsfield, D. R. Mason, A. Schleife, E. W. Draeger, and A. A. Correa, Phys. Rev. Lett. 116, 043201 (2016).

9. E. Holmström, A. Kuronen, and K. Nordlund, Phys. Rev. B 78, 045202 (2008).

10. E. Holmstrm, K. Nordlund, and A. Kuronen, Physica Scripta 81, 035601 (2010).

11. A. V. Krasheninnikov, Y. Miyamoto, and D. Tománek, Phys. Rev. Lett. 99, 016104 (2007).

12. A. Ojanperä, A. V. Krasheninnikov, and M. Puska, Phys. Rev. B 89, 035120 (2014).

13. M. P. Allen and D. J. Tildesley, Computer Simulation of Liquids (Oxford University Press, Oxford, England, 1989).

14. K. Nordlund, J. Wallenius, and L. Malerba, Nucl. Instr. Meth. Phys. Res. B 246, 322 (2005).

15. F. H. Stillinger and T. A. Weber, Phys. Rev. B 31, 5262 (1985).

16. C. A. J. O'Hare, A. M. Green, J. Billard, E. Figueroa-Feliciano, and L. E. Strigari, Phys. Rev. D92, 063518 (2015), arXiv:1505.08061 [astro-ph.CO] .

17. G. Duda, A. Kemper, and P. Gondolo, JCAP 0704, 012 (2007), arXiv:hep$\mathrm{ph} / 0608035$ [hep-ph] .

18. N. Bozorgnia, G. B. Gelmini, and P. Gondolo, Phys. Rev. D84, 023516 (2011), arXiv:1101.2876 [astro-ph.CO] . 